\title{
Comprehensive Meta-Analysis of Maize QTLs Associated With Grain Yield, Flowering Date and Plant Height Under Drought Conditions
}

\author{
Songtao Liu ${ }^{1,2, \dagger}$, Tinashe Zenda ${ }^{1,2, \uparrow}$, Xuan Wang ${ }^{1,2, \uparrow}$, Guo Liu ${ }^{1,2}$, Hongyu Jin ${ }^{1,2}$, Yatong Yang ${ }^{1,2}$, \\ Anyi Dong ${ }^{1,2} \&$ Huijun Duan ${ }^{1,2}$ \\ ${ }^{1}$ Department of Crop Genetics and Breeding, College of Agronomy, Hebei Agricultural University, Baoding, \\ China \\ ${ }^{2}$ North China Key Laboratory for Crop Germplasm Resources of the Education Ministry, Hebei Agricultural \\ University, Baoding, China \\ Correspondence: Huijun Duan, Department of Crop Genetics and Breeding, College of Agronomy, Hebei \\ Agricultural University, Baoding, 071001, China. Tel: 86-1393-1276-716. E-mail: hjduan@hebau.edu.cn
}

Received: March 25, 2019

Accepted: April 27, $2019 \quad$ Online Published: June 15, 2019

doi:10.5539/jas.v11n8p1

URL: https://doi.org/10.5539/jas.v11n8p1

\section{$\dagger$ These authors contributed equally to this work.}

The research was financed by the National Key Research and Development Project of China (Selection and Efficient Combination Model of Wheat and Maize Water Saving, High Yield and High Quality Varieties) [Grant No. 2017YFD0300901].

\begin{abstract}
Drought remains the primary abiotic constraint to maize (Zea mays L.) productivity globally. Maize drought response involves several regulatory quantitative traits and complex gene networks. Therefore, precise location of drought-related quantitative trait loci (QTL) is imperative for drought tolerance breeding. Despite numerous studies identifying several drought-related maize QTLs, some QTL from particular genetic backgrounds showed smaller effects or could not be identified at all in different backgrounds, affected by marker sets, experimental design, mapping populations and statistical methods. Herein, therefore; using 457 published maize QTLs conferring for 18 traits, we have performed meta-analysis of data from various experiments to obtain meta-QTL (MQTL), integrate these fruitful QTL and to mine candidate genes related to drought. Resultantly, 24 MQTL with confidence interval $(\mathrm{CI})<5 \mathrm{~cm}$ were identified to be hot regions. Additionally, 47 drought related gene loci were observed and several candidate genes of the hot MQTL were reorganized by bioinformatics techniques. Thirteen gene (sod4, taf1, rps 1, nthr3, oc13, bas, apx1, asn4, pck2, nac1, gst2, aol and kch4) loci of hot MQTL regions were homologous to their corresponding gene sequences from the PlantGDB database (http://www.plantgdb.org/search/). Further, we used a comparative genomics approach to identify the homologous regions of MQTL in rice (Oryza sativa Japonica) database (http://www.gramene.org) and observed that drought-related rice gene ATG6 was homologous to maize candidate genes GRMZM2G027857_T01 and GRMZM2G027857_T02. Conclusively, our identified MQTLs with narrowed CI could be useful for marker-assisted selection and the candidate genes harnessed for maize drought tolerance breeding.
\end{abstract}

Keywords: Zea mays L., drought stress, marker-assisted selection, meta-analysis, QTL, candidate genes

\section{Introduction}

Maize (Zea mays L.) is the third most important cereal in the world after wheat (Triticum aestivum L.) and rice (Orzya sativa L., Golam et al., 2011). The continued rise in world human population and consequent food demands against the backdrop of worsening global climate change has prompted greater need for the crop (Xu et al., 2014; Maazou et al., 2016). However, as a result of the continuing climate change phenomenon, drought stress will remain the primary environmental limitation to maize productivity (Rao et al., 2016). The maize crop is most susceptible to drought stress at the reproductive stage (Tuberosa, 2012). Therefore, maize breeders have the burden of developing drought resilient crop varieties in order to sustain higher yields and global food security under the ravaging climate change conditions. 
Drought tolerance breeding has remained a very tedious task because of the high variability of drought stress and insufficient understanding of its complexity (Hao et al., 2010). Maize drought response involves several regulatory quantitative traits and complex gene and metabolic networks (Li et al., 2010; Adebayo et al., 2014). Quantitative trait loci (QTL) analysis of maize drought tolerance involves various aspects, including morphological traits (such as plant height, ear position, and branches per tassel etc.), root, physiological, and biochemical related traits (ABA, degree of leaf senescence, photosynthesis parameters, protein content, stomatal conductance, osmotic adjustment etc.), and yield traits (ear length, 100-kernel weight, ears per plant, and grain yield etc.) among others (Lu et al., 2006; Edmeades, 2013).

Since increasing yield potential under water-stressed conditions is the first target of all breeding programs, the identification of QTL for grain yield and its components becomes crucial for all QTL studies (Luo et al., 2006; Adebayo et al., 2014). The main indicators for identification of yield traits include number of ears per plant, ear weight, number of grains per ear, 100-grain weight and grain yield (Shin et al., 2015). Amongst all these, grain yield can best reflect the effect of drought stress (Edmeades et al., 1999). In addition, morphological characters have a close relationship with plant water absorption and loss. These include a well-developed root system, leaf morphology, plant type and anthesis-silking interval (Hall et al., 1982; Ribaut et al., 2009).

Whereas drought stress might occur throughout the maize growing period whenever the crop is grown without irrigation, flowering period is the most susceptible to drought stress (Tuberosa et al., 2002). Under water-limited conditions, ear growth slows, eventually retarding silk emergence. Consequently, the length of the ASI increases significantly, which may lead to complete abortion of ears and the plant becomes barren (Maazou et al., 2016). Thus, maize drought tolerance is a consequence of coordination amongst morphological, physiological and biochemical characters, and will also depend on genotypes (Li et al., 2010). Additionally, maize drought tolerance related agronomic traits are not only influenced by external environment, but also controlled by a vast array of genes with minor effects. To this end, precise quantitative trait loci (QTL) identification becomes the focal point when researchers apply molecular biology to study the trait (Arcade et al., 2014).

Over the previous two decades, a mounting body of literature underlying yield-related, plant type, root-related and physiological traits have been published; the number of identified QTL on all maize chromosomes (Chr) related to water deficit tolerance has increased significantly (Hao et al., 2010). However, there is an obvious issue that few identical QTL were identified even in the same environment in the same experimental population by different researchers or different years (Li et al., 2010). Moreover, most data showed that partly or wholly non-overlapping QTL are affected by different combinations and/or experiments conducted in different environments (Orf et al., 1999; Rong et al., 2007). Since maize drought tolerance is under highly complex genetic control, it is difficult to manipulate, making it hard to predict the usefulness of QTL for marker-assisted selection (MAS). The integration of QTL data from diverse resources to compare their locational position consistence and specifying co-locations with candidate genes is therefore imperative for selecting QTL for breeding applications (Hanocq et al., 2007). Despite numerous studies identifying several drought-related maize QTLs, some QTL from particular genetic backgrounds showed smaller effects or could not be identified at all in different backgrounds, affected by marker sets, experimental design, mapping populations and statistical methods (Li et al., 2010). The lack of repeatability of QTL effects across different populations and across environments limits the use of this kind of QTL for MAS by plant breeders. Further, it is very difficult to identify the functional drought-tolerant genes and then develop effective molecular markers inside them (Hao et al., 2010). Therefore, integrating these 'initial' QTL, developing a consensus map, and exploiting consensus QTL and their underlying genes becomes a crucial short cut to assist breeding activities.

Meta-analysis is a statistical analysis that combines data from different sources in a single study, and can overcome the limits of individual studies by analysing generally all practical data to achieve a more informative and truthful conclusion (Wang et al., 2006). It combines data from independent researches to identify consensus QTL across studies, validate QTL effects from across environments or genetic backgrounds and to refine QTL positions on the consensus map (Rong et al., 2007; Swamy et al., 2011). This method has been proved an efficient way for use in maize (Goffinet \& Gerber, 2000), wheat (Wang et al., 2016), soybean (Wang et al., 2017), grasses (Swamy et al., 2011), potato (Danan et al., 2011) and rice (Courtois et al., 2009). Although QTL and other loci gathered from multiple maps remain a manual and tedious task, this is a critical step to reveal co-locations between genes and QTL. To take advantage of QTL meta-analysis method in maize breeding, several researchers are aiming at diverse agronomic characters by integrating the results from different experiments. For instance, Xiang and co-workers (2012) integrated 241 QTL for correlations of ear rot (ER) and grain moisture content (GM) from 29 studies in maize and observed 29 MQTL and 44 MQTL for ER and GM, respectively chromosomally located differently. Thus, meta-analysis has been widely used in map integration in 
crops such as wheat (Hanocq et al., 2007), maize (Truntzler et al., 2010), grasses (Swammy et al., 2011) and cotton (Lacape et al., 2010).

Discovering genes involved in the quantitative traits is the first step in understanding biological processes underlying these traits. QTLs often have long confidence intervals (CIs), therefore, it is difficult to map genes related to agronomic traits (Semagn et al., 2013). Fortunately, meta-analysis could narrow down the CI of integrated QTLs to increase about twofold precision in estimation of QTL position compared to the initial QTL position of the corresponding region (Goffinet \& Gerber, 2000).

In the present study; therefore, we used QTL meta-analysis strategy to integrate and reanalyze QTL for three agronomically important traits in maize; including yield, plant height and flowering time, under drought stressed conditions. Additionally, we developed a consensus map and identified consensus QTL for these traits, and have provided MTQL markers with high effects and narrowed confidence intervals $(<5 \mathrm{~cm})$ for possible employment in marker assisted selection or fine-mapping QTL for gene mining. Moreover, we used some bioinformatics tools to dig-out some candidate genes responding to drought stress that were harboured in diverse chromosomal regions. Further, we used a comparative genomics approach to identify the homologous regions of MQTL in rice (Oryza sativa Japonica). We hope that several promising MQTL and candidate genes identified herein could be useful for MAS and maize drought tolerance breeding.

\section{Method}

\subsection{Maize Quantitative Trait Loci (QTL) Data Collection}

In this study, we surveyed the QTLs detected under water-deficit condition or under both water-sufficient and water-deficit conditions. We used twenty-two studies on maize yield and yield-related traits (yield, kernel weight, kernel row number, kernel number per ear, kernel number per row, ear diameter, ear length, ear weight, kernel length, kernel width; published from 1994 to 2017), seventeen reports on flowering-date-related traits (male flowering time, female flowering time and anthesis-silking interval; published from 1994 to 2017), and fourteen researches on maize plant-type-related traits (plant height, ear height; published from 1994 to 2012) (Table 1). The English and Chinese literature were retrieved from the PubMed (http://www.ncbi.nlm. nih.gov/pubmed), and the China Knowledge Resource Integrated Database (CNKI; http://www.cnki.net) and Baidu Scholar (http://xueshu.baidu.com), respectively. The information including QTL names, map names, traits, likelihood of odd (LOD) score, proportion of variance explained $\left(\mathrm{R}^{2}\right)$, marker and linkage group, QTL position, "QTL from" and "QTL to" was then reorganized.

\subsection{Refinement of Maize QTLs}

In QTL position refinement procedure, the most important parameter is each QTL map position (most likely position and confidence interval) and the proportion of phenotype. When a QTL position was not available in the paper, the $95 \%$ confidence interval was calculated according to Darvasi and Soller (1997) using the following formulae:

$$
\begin{aligned}
& \mathrm{CI}=530 /\left(\mathrm{NR}^{2}\right) \\
& \mathrm{CI}=163 /\left(\mathrm{NR}^{2}\right)
\end{aligned}
$$

where, Formular 1 was suitable for both backcross population and F2 population, and formular 2 was suitable for recombinant inbred line (RIL) population. $\mathrm{CI}$ is the confidence interval of a QTL, N is the population size, and $\mathrm{R} 2$ is the phenotypic contribution of a QTL.

\subsection{Consensus Map Construction}

It is vital to verify connectivity between input maps before performing the construction of the consensus map (Hanocq et al., 2007). In order to list markers which were not consistent in the different individual maps, in the current study, we used the command "InfoMap" of the BioMercator 4.2 software (http://www.mybiosoftware. com/biomercator-2-1-genetic-maps-qtl-integration.html) (URGI, Versailles, France). For each linkage group, InfoMap display some descriptive statistics about the marker maps to determine if the input data can be used for creating a consensus map. Then, we used the "ConsMap" command of the MetaQTL software BioMercator V4.2 (URGI, Versailles, France) to create a consensus marker map. The method implemented in MetaQTL is based on a Weighted Least Square (WLS) strategy (Arcade et al., 2014). For this analysis, the population size and the type are necessary (in the input data) to determine the accuracy. We used the high-density genetic linkage map IBM2 2008 Neighbors (Intermated B73 $\times$ Mo17 Map) as reference map, which covered $8054.28 \mathrm{~cm}$ and consisted of 
15991 markers. The original maps of QTL collected from different researches (which used different backgrounds, populations and methods) were compared with the reference map to generate a consensus map.

Table 1. Bibliography of Quantitative Trait Loci (QTL) used in this study

\begin{tabular}{|c|c|c|c|c|c|c|c|}
\hline Parent lines & $\begin{array}{l}\text { Population } \\
\text { size }\end{array}$ & $\begin{array}{l}\text { Population } \\
\text { type }\end{array}$ & Environment & Marker type & $\begin{array}{l}\text { Analytical } \\
\text { method }\end{array}$ & Traits & Reference \\
\hline Mo17×H99 & 150 & $F 2: 3$ & 2 & RFLP & CIM & GY KW EN EL ED CD KD KR & Veldboom \& Lee (1996) \\
\hline $\mathrm{SD} 34 \times \mathrm{SD} 35$ & 120 & F3 & 2 & RFLP & IM & GY EN ASI & Agrama \& Moussa (1996) \\
\hline Ac7643S5×Ac7729/TZSRWS5 & 234 & F3 & 2 & AFLP & IM and CIM & MF FF ASI & Ribaut et al.(1996) \\
\hline Ac7643S5×Ac7729/TZSRWS5 & 234 & F3 & 2 & AFLP & IM and CIM & GY KN EN & Ribaut et al. (1997) \\
\hline Ki3 $\times$ CML139 & 472 & $\mathrm{~F} 2$ & 2 & RFLP & CIM & ASI FF FH & Khairallah et al. (1998) \\
\hline B73×H99 & 142 & RIL & 2 & RFLP, SSR and AFLP & IM & MF FF ASI FH & Sari-Gorla et al. (1999) \\
\hline B73×H99 & 142 & RIL & 2 & RFLP, SSR and AFLP & IM & EN EL KN EW KW & Frova et al. (1999) \\
\hline Lo964×Lo1016 & 171 & F3 & 2 & RFLP & IM & GY & Tuberosa et al. (2002) \\
\hline Huangzao4×Ye107 & 184 & $\mathrm{~F} 2: 3$ & 2 & SSR & CIM & ASI GY ES & Li et al. (2003) \\
\hline N87-1×9526 & 183 & $\mathrm{~F} 2: 3$ & 2 & SSR & CIM & FH ASI GY EH MF FF & Gao (2004) \\
\hline A118×91huang15 & 139 & $\mathrm{~F} 2: 3$ & 2 & SSR & IM & FH EH MF FF ASI GY & $\mathrm{Wu}(2005)$ \\
\hline $\mathrm{X} 178 \times \mathrm{B} 73$ & 234 & $\mathrm{~F} 2$ & 2 & SSR & CIM & $\begin{array}{l}\text { MF FF ASI KN EW EN } \\
\text { CW GY KW FH EH }\end{array}$ & Xiao (2005) \\
\hline N87- $1 \times 9526$ & 183 & $\mathrm{~F} 2: 3$ & 2 & SSR & CIM & GY & Gao et al. (2005a) \\
\hline N87- $1 \times 9526$ & 183 & $\mathrm{~F} 2$ & 2 & SSR & CIM & ASI FF MF & Gao et al. (2005b) \\
\hline Zong $3 \times 87-1$ & 221 & RIL & 2 & SSR & CIM & EL KN KW & Lu et al. (2006) \\
\hline $5003 \times \mathrm{p} 138$ & 450 & RIL & 2 & SSR & CIM & FH GY & Jiang (2006) \\
\hline D5×7924 & 180 & F5:6 & 4 & SSR & CIM & EH FH GY ES ASI & Zhu (2008) \\
\hline Huangzao $4 \times$ Ye 478 & 235 & $\mathrm{~F} 2: 3$ & 2 & SSR & CIM & $\mathrm{FH}$ & Wang (2008) \\
\hline N87-1×9526 & 183 & $\mathrm{~F} 2: 4$ & 2 & SSR & CIM & GY ASI FH & Fu et al. (2008) \\
\hline B73×H99 & 142 & RIL & 2 & RFLP, SSR and AFLP & $\mathrm{IM}$ & MF FF ASI FH EL EW KW KN & Marino et al. (2009) \\
\hline CML444×SC-Malawi & 236 & RIL & 4 & RFLP and SSR & CIM & KW GY KN MF ASI FH & Messmer et al. (2009) \\
\hline Huangzao4×Qi319 & 230 & $\mathrm{~F} 2: 3$ & 4 & SSR & CIM & GY KN KW KR EL ED CD EW & Peng et al. (2010) \\
\hline D5 $\times 7924$ & 180 & $\mathrm{~F} 2: 3$ & 2 & SSR & CIM & EH FH GY ES ASI & Zhu et al. (2011) \\
\hline D5 $\times 9381$ & 215 & F $3: 4$ & 2 & SSR & CIM & GY ASI EL ED FH KN & Wang (2011) \\
\hline Lin1×Xiang97-7 & 160 & $\mathrm{~F} 2: 3$ & 4 & SSR & CIM & ASI KW GY FH & Chen et al. (2012) \\
\hline Chang7-2×TS141 & 218 & $\mathrm{~F} 2: 3$ & 4 & SSR & CIM & $\mathrm{EN}$ & Zhao et al. (2017) \\
\hline Langhuang $\times$ TS141 & 202 & $\mathrm{~F} 2: 3$ & 4 & SSR & CIM & EN & Zhao et al. (2017) \\
\hline $\mathrm{X} 178 \times \mathrm{B} 73$ & 234 & $\mathrm{~F} 2: 3$ & 6 & SSR and AFLP & IM and CIM & ES GY ASI & Hao et al. (2017) \\
\hline
\end{tabular}

Note. (Population type: RIL = recombinant inbred line; F2:3, F2:4, F3:4, F5:6 = segregating populations at different stages); Environment: number of geographical locations; (Marker type: SSR = simple sequence repeat, RFLP $=$ restriction fragment length polymorphism, AFLP = amplified fragment length polymorphism); (Analytical method: IM = interval mapping, $\mathrm{CIM}=$ composite interval mapping); (Traits: $\mathrm{FH}=$ plant height; $\mathrm{EH}$ = ear height; $\mathrm{ASI}=$ anthesis-silking interval; $\mathrm{FF}=$ female flowering; $\mathrm{GY}=$ grain yield; $\mathrm{KW}=$ kernel weight; $\mathrm{EN}$ = ear number; $\mathrm{EL}=$ ear length; $\mathrm{ED}=$ ear diameter; $\mathrm{CD}=$ cob diameter; $\mathrm{KD}=$ kernel diameter; $\mathrm{KR}=\mathrm{kernel}$ row number; $\mathrm{ES}=$ ear-setting; $\mathrm{BIO}=$ biomass; $\mathrm{CW}=$ cob weight).

\subsection{QTL Projection and Meta-Analysis}

We created a consensus map and QTL of individual experiments were projected based on the common marker between the original map and reference map by means of a homothetic function described by Chardon et al. (2004). Some controversial markers between original and reference maps were deleted to make sure the analysis was accurate. When the consensus map was built, meta-analysis was used to determine the existence of the consensus QTL and locate the confidence interval (CI). Map projection and QTL meta-analysis were performed using the BioMercator 4.2 software. Although the software has been given five models, the lowest Akaike Information Criterion (AIC) value is believed to be optimum. In the model, the position of each QTL in LG decided the consensus QTL position. The formular for meta-analysis was according to Goffinet and Gerber (2000) as follows:

$$
\operatorname{var}(Q T L)=1 / \Sigma 1 / \sigma_{\mathrm{i}}^{2}
$$

where, $\sigma_{\mathrm{i}}$ is the position of the variance for each of the QTLs on the LG. A 95\% CI of the consensus QTL was calculated from the var (QTL):

$$
C I=3.92 \times \operatorname{var}(Q T L)^{1 / 2}
$$


The AIC value depends on the simulation of each model. The smallest AIC model is close to the consensus QTL. The mean R2 values of the original QTLs in the region were the explained variance of this consensus QTL. In this study, the MQTL with less than three original QTLs were discarded.

\subsection{Meta-QTL (MQTL) Interval Mapping of Candidate Genes Under Drought Stress}

The physical locations of meta-QTL (MQTL) were estimated with the help of MaizeGDB (https://maizegdb.org) and GRAMENE (http://www.gramene.org) web servers. The B73 RefGen_v2 sequence information of maize physical map was integrated with the marker information of IBM2 2008 linkage map which could detect the approximate location of given markers or genes on the B73 RefGen v3. Then, we downloaded the candidate gene sequences of the MQTL interval through online batch download tool "Download Region Data" in PlantGDB database (http://plantgdb.org). In this study, we only identified annotated transcripts in MQTL with < $5 \mathrm{Mb}$ interval.

\subsection{Discovery of Drought-Related Gene Loci (and Candidate Genes) in Maize MQTL}

Firstly, we reorganized the MQTL interval and adjacent area of the drought related gene loci of the reference map IBM2 2008 Neighbors, retrieved from the NCBI (http://www.ncbi.nlm.nih.gov) web server according to the related gene loci for obtaining sequence information. We downloaded the protein sequences of the MQTL interval through online batch download tool "Download Region Data" in PlantGDB database. Then, we analyzed the conserved domains of all protein sequences using online batch 'Search tool' in website Pfam (http://pfam.sanger.ac.uk). Lastly, the results of these analyses were compared and integrated with the genetic annotation information provided by the NCBI website to dig out drought related genes of MQTL interval and adjacent area.

\subsection{Extraction of Drought Related Genes in Maize Based on Gene Comparative Mapping}

Drought related genes were searched from the rice genome database Gramene (http://www.gramene.org) and mapped to the maize IBM2008 linkage map by the "Cmap" function of the GRAMENE website. Then, we downloaded the protein sequences of the MQTL intervals through online batch download tool "Download Region Data" in PlantGDB database. To dig out drought related genes in maize which were homologous to rice, we analyzed the conserved domains of the downloaded and predicted maize gene/protein sequences by comparing them to the rice drought-related gene/protein sequences through the use of "batch search function" of the Pfam (http://pfam.xfam.org) website.

\section{Results}

\subsection{Summary of QTL Information Collected}

A total of 457 QTLs for maize yield, plant morphology, and florescence under drought stress condition were compiled from 28 published papers and 20 populations. These QTLs were associated with 18 traits categorized into three types, including grain yield, flowering date, and plant morphology (height). Amongst the 457 QTL, 207 from 22 researches and 18 populations were related to grain yield, 130 from 17 published papers and 12 populations were related to flowering date, whereas 94 from 14 papers and 11 populations were related to plant morphology. Population size ranged from 120 to 472, including F2, F3, F2:3, F3:4 and recombinant inbred lines (RILs) populations (Table 1).

\subsection{QTL Number and Distribution on Maize Chromosomes}

Our results showed that the collected QTLs were non-uniformly distributed in all the ten maize chromosomes; QTL on Chr 1, 2, 3, 5 and 9 were more uniformly distributed amongst all of them. QTL number was highest (up to 83 QTL) on Chr 1 and lowest (29) on Chr 7. Meanwhile, with regards to yield and flowering date related traits, QTL were mostly found on Chr 1, whereas pertaining to plant morphology related traits, the QTL were almost uniformly distributed across all ten maize chromosomes. The trait of anthesis-silking interval, with 70 QTL, was the highest among all the traits (Table 2). 
Table 2. Summary of QTLs associated with drought tolerance in maize

\begin{tabular}{lllllllllllll}
\hline Trait & Trait & \multicolumn{1}{l}{ Chr1 } & Chr2 & Chr3 & Chr4 & Chr5 & Chr6 & Chr7 & Chr8 & Chr9 & Chr10 & QTL number \\
\hline \multirow{5}{*}{ Yield-related } & Kernel weight & 6 & 4 & 4 & 5 & 3 & 3 & 4 & 2 & 2 & 4 & 37 \\
& Ear diameter & 1 & 1 & - & - & 2 & 1 & 1 & 1 & - & - & 7 \\
& Ear length & 2 & 2 & 2 & - & 2 & 1 & - & 1 & 1 & 1 & 12 \\
& Ear number & 6 & 2 & 3 & - & 1 & 4 & 1 & - & 2 & 1 & 20 \\
& Ear weight & 1 & 2 & - & - & - & - & - & - & 2 & 2 & 7 \\
& Ear-setting & 3 & 1 & 3 & - & 1 & 1 & - & 1 & 3 & 2 & 15 \\
& Grain yield & 10 & 1 & 7 & 8 & 9 & 6 & 2 & 4 & 3 & 7 & 57 \\
& Kernel diameter & 1 & 1 & 1 & - & - & 1 & - & - & - & - & 4 \\
& Kernel number & 4 & 2 & 1 & 3 & 3 & - & 1 & 2 & 4 & 3 & 23 \\
& Kernel row number & - & 1 & 2 & 1 & 2 & 1 & - & - & - & - & 7 \\
& Cob diameter & 2 & 2 & - & - & 2 & - & 1 & 1 & - & - & 8 \\
& Cob weight & 1 & - & 1 & - & 1 & - & 1 & - & 2 & - & 6 \\
\multirow{5}{*}{ Flowering date } & Biomass & 1 & - & - & - & - & - & 1 & 2 & - & - & 4 \\
& Male flowering & 9 & 7 & 4 & 5 & 4 & 3 & 2 & 3 & 4 & 2 & 43 \\
$\cdots$ & Female flowering & 7 & 6 & 3 & 1 & 2 & 4 & 5 & 6 & 6 & 3 & 43 \\
& Anthesis-silking interval & 16 & 9 & 13 & 3 & 6 & 4 & 3 & 5 & 7 & 4 & 70 \\
\hline \multirow{5}{*}{ Plant type } & Plant height & 7 & 9 & 2 & 7 & 3 & 5 & 6 & 10 & 7 & 2 & 58 \\
& Ear height & 6 & 3 & 4 & 5 & 4 & 6 & 1 & 2 & 1 & 4 & 36 \\
& Total & 83 & 53 & 50 & 38 & 45 & 40 & 29 & 40 & 44 & 35 & 457 \\
\hline
\end{tabular}

Note. $\mathrm{Chr}=$ chromosome.

\subsection{Constructed QTL Consensus Map and MQTL Identified by Meta-analysis}

The initial map was projected onto the reference map by BioMecrcator 4.2 software and the consensus map constructed. The initial markers of the initial map on Chr 4 and 10 failed to connect with other maps and we discarded them in constructing the consensus map [31]. A total of 233 QTL from 22 studies were short-listed for grain yield and its components under drought stress. After deleting the QTL with likelihood of odd (LOD) value $<2$ and phenotypic variance $<5$, there were 220 QTLs projected onto a consensus map. The chromosomal regions with $<3$ original QTLs were not considered for MQTL. Thus, a total of 37 MQTL covering 10 maize chromosomes were identified based on the lowest Akaike Information Criteion (AIC) values criteria (Hao et al., 2010). Each Chr contained an average of 2 to 6 MQTL. There were 6 MQTL on Chr 1; 3 MQTL on Chr 3, 7, 8 and 9; 4 MQTL on Chr 2, 4 and 6; 5 MQTL on Chr 5; and 2 MQTL on Chr 10 (Table 3; Figure S1). Similar to the collected QTL distribution, MQTL on Chr 1 was the highest. Of the identified 37 MQTL, 13 initial QTLs were condensed on Chr 10 with the map genetic position of $117.4 \mathrm{~cm}$, which explained $9.69 \%$ phenotypic variance and narrowed the confidence intervals to $2.44 \mathrm{~cm}$. Twelve initial QTLs were condensed on $\mathrm{Chr} 1 \mathrm{with}$ the map genetic position of $208.46 \mathrm{~cm}$ and mean phenotypic variance of more than $10 \%$ (reaching up to $12.33 \%$ ). These MQTLs were designated sequentially from MQTL 1 to MQTL 37 according to their Chr locations (Table 3; Figure S1).

A total of 140 flowering time related traits were collected from 17 studies and were projected onto the reference map; 133 QTL were successfully projected on all the 10 maize chromosomes. Maximum (25) QTLs were on Chr 1. In this part, 28 MQTL related to maize flowering date were identified. The number of MQTL on each chromosome ranged from 1 to 5 . Chromosome 1 had the most number of MQTL, similar to the results of meta-analysis on grain yield related traits (Table 3; Figure S1). The phenotypic variance of the MQTL varied from $6 \%$ to $34.67 \%$, with the confidence interval ranging from $0.69 \mathrm{~cm}$ to $31.33 \mathrm{~cm}$. Meanwhile, MQTL51 on Chr 7 had minimum CI of $0.69 \mathrm{~cm}$ and corresponded to 4 initial QTLs, with an average R2 of $8.75 \%$. MQTL42, MQTL46, MQTL47, MQTL58 and MQTL62 on Chr 1, 3, 3, 8 and 9, respectively corresponded to 3, 6, 3, 6 and 4 initial QTL, with R2 ranging from $6.5 \%$ to $18.75 \%$. The location, confidence interval, phenotypic variance and flanking markers information of each MQTL are provided in Table 3.

A total of 94 QTLs collected from 14 researches were associated with plant height and ear height, and were distributed on all the ten chromosomes. Among these, 4 QTL could not be projected onto the reference map 
because they did not have common markers with the reference map or genetic linkage map. The remaining 90 initial QTL that were projected onto all 10 chromosomes ranged from 5 to 12 QTL on each Chr. Chromosome 9 had the least (5), whereas Chr 8 had the largest (16) number of QTL. In this part, a total of 13 MQTL were distributed on eight chromosomes, with the exception of Chr 3 and 7 (Figure S1). The MQTL of Chr 3 and 7 were discarded due to less than three initial QTL projected on them. There were 2 MQTL on each of Chr 1, 2 and 5; whereas Chr 4, 6, 9 and 10 contained 1 MQTL each. Meanwhile, on Chr 8, sixteen initial QTL were aggregated to 3 MQTL and positioned at $68.68 \mathrm{~cm}, 119.05 \mathrm{~cm}$ and $152.36 \mathrm{~cm}$, with the confidence interval narrowed to $6.63 \mathrm{~cm}, 3.90 \mathrm{~cm}$ and $8.47 \mathrm{~cm}$, respectively. The initial QTL of each MQTL ranged from 3 to 6 , with the $\mathrm{R} 2$ ranging from $6.0 \%$ to $29.0 \%$ (Table 3 ).

Table 3. Meta-QTLs for yield, flowering date and plant type related traits under drought stress conditions identified by meta-analysis

\begin{tabular}{|c|c|c|c|c|c|c|c|c|c|c|}
\hline Trait & Meta-QTL & $\mathrm{Chr}$ & AIC Value & $\begin{array}{l}\text { Position of } \\
\text { MQTL }\end{array}$ & $\mathrm{CI}$ & $\begin{array}{l}\text { No of } \\
\text { initial QTL }\end{array}$ & $\begin{array}{l}\text { Mean } \mathrm{R}^{2} \text { for } \\
\text { initial QTL }\end{array}$ & $\begin{array}{l}\text { Flanking markers } \\
\text { of the position }\end{array}$ & Interval of physical map & Related gene loci \\
\hline \multirow{35}{*}{ 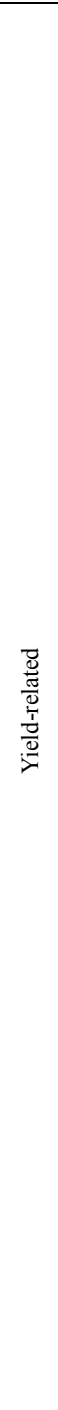 } & MQTL1 & 1 & 281.62 & 207.88 & 7.04 & 10 & 13.4 & IDP2420-TIDP4556 & $4,758,454-4,767,124$ & \\
\hline & MQTL2 & & & 222.37 & 4.11 & 12 & 11.7 & gpm774d-TIDP8795 & $21,419,789-22,381,090$ & tip rpe1 \\
\hline & MQTL3 & & & 235.7 & 4.88 & 7 & 11.29 & IDP8569-IDP8612 & $60,047,657-60,678,493$ & dvr1 $\operatorname{sod} 4$ \\
\hline & MQTL4 & & & 253.04 & 5.91 & 5 & 18.4 & w18-umc 2047 & $266,933,205-263,011,985$ & \\
\hline & MQTL5 & & 99.34 & 174.92 & 4.15 & 7 & 7.57 & umc11-umc53b & $21,419,789-22,003,995$ & k1s2 \\
\hline & MQTL6 & 2 & 106.61 & 72.4 & 11.2 & 5 & 11.2 & umc 259 b-bzip 121 & $31,889,777-34,239,593$ & \\
\hline & MQTL7 & & & 83.74 & 2.86 & 5 & 9.2 & IDP2468-umc1454 & $63,944,183-69,704,680$ & mde1 \\
\hline & MQTL8 & & 66.4 & 122.74 & 8.49 & 3 & 13.33 & IDP7614-csu64a(grf) & $224,335,836-224,605,071$ & \\
\hline & MQTL9 & & & 140.32 & 9.23 & 5 & 12.6 & IDP2512-npi294a & $232,489,953-232,824,897$ & \\
\hline & MQTL10 & 3 & 233.9 & 35.62 & 4.7 & 7 & 12.86 & TIDP3332-npi71 & $1,713,115-2,270,923$ & npi71 \\
\hline & MQTL11 & & & 57.7 & 3.96 & 7 & 11.71 & pza00749-IDP9134 & $9,843,447-10,080,572$ & pld1 tip4 myb40 \\
\hline & MQTL12 & & & 80.59 & 3.75 & 8 & 9.75 & umc165a-bnl6.16a & $176,248,449-191,054,985$ & gst8 got1 taf1 \\
\hline & MQTL13 & 4 & 150.63 & 61.39 & 2.64 & 7 & 10 & bnlg1217-pza03385 & $41,977,452-42,016,246$ & su1 fie1 \\
\hline & MQTL14 & & & 100.21 & 6.51 & 5 & 11.4 & umc2365-umc1051 & $189,494,506-193,438,448$ & \\
\hline & MQTL15 & & & 125.43 & 10.07 & 4 & 12 & gpm350b-gpm87a & $237,822,149-239,027,984$ & \\
\hline & MQTL16 & 5 & 39.68 & 80.12 & 5.24 & 3 & 21 & pza00818-IDP3834 & $1,080,009-1,538,562$ & \\
\hline & MQTL17 & & & 98.96 & 10.46 & 4 & 12.25 & umc40-umc1679 & $87,290,927-3,429,663$ & \\
\hline & MQTL18 & & 165.25 & 136.43 & 4.41 & 5 & 8.8 & IDP4864-bcd207b & $20,922,705-23,141,360$ & \\
\hline & MQTL19 & & & 151.53 & 3.48 & 7 & 8.57 & bnl10.12-umc1264 & $175,145,318-175,677,720$ & rps27b \\
\hline & MQTL20 & & & 196.97 & 19.97 & 4 & 6.25 & umc1792-bnlg1695 & $218,451,914-221,681,657$ & \\
\hline & MQTL21 & 6 & 125.01 & 225.4 & 5.69 & 3 & 26.33 & TIDP3537-mmp160 & $87,122,202-66,438,133$ & \\
\hline & MQTL22 & & & 253.53 & 7.22 & 5 & 8 & gpm250b-IDP4314 & $134,804,842-142,486,587$ & \\
\hline & MQTL23 & & & 268.86 & 10.5 & 4 & 14.25 & gpm631-roa1 & $153,555,627-154,285,844$ & \\
\hline & MQTL24 & & & 281.52 & 5.77 & 3 & 35 & umc237-umc 1490 & $161,024,525-162,276,992$ & \\
\hline & MQTL25 & 7 & 96.07 & 50.05 & 7.01 & 3 & 12.67 & TIDP4658-csu251a & $4,549,325-4,816,431$ & \\
\hline & MQTL26 & & & 66.28 & 4.09 & 5 & 10.8 & gpm804-rps1 & $14,539,980-17,234,112$ & rps15 rps1 taf1 \\
\hline & MQTL27 & & & 78 & 4.78 & 4 & 12 & umc1660-umc2630 & $141,594,488-151,703,776$ & nthr3 oc13 nac1 \\
\hline & MQTL28 & 8 & 168.68 & 85.16 & 15.81 & 5 & 8.2 & gpm934-IDP9127 & $20,305,710-20,699,697$ & \\
\hline & MQTL29 & & & 122.68 & 7.59 & 6 & 10.17 & umc2357-mmp64 & $170,061,917-170,127,656$ & \\
\hline & MQTL30 & & & 132.22 & 3.83 & 6 & 11.5 & obf4-npi315a & $171,334,858-175,442,935$ & psei2 \\
\hline & MQTL31 & 9 & 122.22 & 102.76 & 6.79 & 3 & 10 & znod1-gpm79a & $16,979,346-18,607,113$ & \\
\hline & MQTL32 & & & 129.93 & 3.9 & 9 & 9.33 & $\operatorname{csu355}(\mathrm{ext})$-polm2 & $131,908,055-154,478,416$ & $\begin{array}{l}\text { vacs1 phyB2 } \\
\text { apx1 asn4 pck2 }\end{array}$ \\
\hline & MQTL33 & & 40.02 & 145.79 & 4.72 & 5 & 15 & pco063085-telomere9L & $149,480,368-156,132,972$ & \\
\hline & MQTL34 & 10 & 176.32 & 117.4 & 2.44 & 13 & 9.69 & mpk6-gpm840a & $84,128,565-88,611,477$ & gst2 rps3 nac1 \\
\hline & MQTL35 & & & 128.05 & 8.05 & 8 & 9.75 & gpm503-IDPCP59559 & $133,899,843-125,630,737$ & \\
\hline \multirow{8}{*}{ 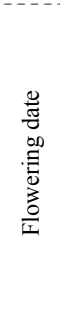 } & MQTL36 & 1 & 196.91 & 206.44 & 22.3 & 3 & 7.67 & mHbrBT90-Mo17-dpr1 & $2,978,024-3,792,043$ & \\
\hline & MQTL37 & & & 242.54 & 8.88 & 8 & 9.67 & npi234-umc1568 & $34,652,377-15,808,040$ & \\
\hline & MQTL38 & & & 272.21 & 9.63 & 8 & 9 & umc2396-gpm260a & $198,104,270-200,547,561$ & \\
\hline & MQTL39 & & 38.52 & 303.15 & 0.24 & 5 & 9 & pza00978-mab18 & $283,187,143-287,192,923$ & ao1 ces 1 \\
\hline & MQTL40 & 2 & 63.08 & 129.77 & 11.54 & 4 & 10.25 & IDP1657-mmc0271 & $202,196,007-198,625,109$ & \\
\hline & MQTL41 & & 84.82 & 47.04 & 5.54 & 3 & 18 & TIDP5226-pco102445 & $5,818,068-6,534,813$ & \\
\hline & MQTL42 & & & 157.67 & 14.9 & 4 & 9.5 & IDP4819-TIDP6098 & $222,275,941-222,468,376$ & \\
\hline & MQTL43 & 3 & 130.27 & 67.31 & 1.82 & 6 & 7.67 & IDP2399-gpm713 & $3,840,177-4,379,610$ & kch4 \\
\hline
\end{tabular}




\begin{tabular}{|c|c|c|c|c|c|c|c|c|c|c|}
\hline \multirow{18}{*}{ 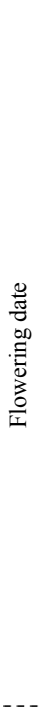 } & MQTL44 & & & 110.27 & 3.16 & 3 & 10 & nrp1-umc92a & $29,838,476-31,245,032$ & nrp1 e4 \\
\hline & MQTL45 & & & 123.21 & 14.44 & 3 & 8.67 & agrp97-tda64 & $136,086,344-137,548,200$ & \\
\hline & MQTL46 & 4 & 74.97 & 132.83 & 11.04 & 6 & 9.33 & gst26-bnl17.09 & $36,299,524-36,460,064$ & \\
\hline & MQTL47 & 5 & 96.45 & 197.49 & 11.5 & 3 & 7.33 & ucsd64a-IDP3964 & $7,052,510-7,265,191$ & \\
\hline & MQTL48 & & & 292.29 & 25.51 & 3 & 6 & umc68-bnl5.24 & $205,440,973-217,012,402$ & \\
\hline & MQTL49 & 6 & 79.13 & 266.21 & 13.63 & 5 & 9.8 & csu116a-csu382a(cld) & $107,401,374-132,064,555$ & \\
\hline & MQTL50 & & & 284.09 & 5.65 & 3 & 34.67 & bnl8.08c-umc 1859 & $154,096,569-154,494,718$ & \\
\hline & MQTL51 & 7 & 122.15 & 81.85 & 0.69 & 4 & 8.75 & IDP4192-TIDP5555 & $124,265,889-129,111,170$ & asc1 \\
\hline & MQTL52 & & & 104.81 & 11.14 & 3 & 15.67 & gpm392-IDP4527 & $153,455,716-153,584,620$ & \\
\hline & MQTL53 & & & 137.63 & 9.49 & 4 & 8 & gpm683b-agrr44 & $165,268,333-166,901,107$ & \\
\hline & MQTL54 & 8 & 123.15 & 120.82 & 1.68 & 6 & 6.5 & bnl17.20-rack2 & $39,115,777-74,024,212$ & prp1 \\
\hline & MQTL55 & & & 151.76 & 11.48 & 4 & 13 & IDP6608-umc1121 & $141,912,596-147,824,187$ & \\
\hline & MQTL56 & & & 190.04 & 8.11 & 3 & 24.67 & npi328a-cdo187 & $165,636,122-166,244,750$ & \\
\hline & MQTL57 & 9 & 143.2 & 73.74 & 13.14 & 3 & 12 & gpm117a-TIDP6487 & $4,382,875-5,538,490$ & \\
\hline & MQTL58 & & & 130.71 & 3.29 & 4 & 18.75 & asg37-IDP8628 & $95,980,842-96,199,121$ & fdh2 mrpa pep1 \\
\hline & MQTL59 & & & 162.13 & 12.51 & 5 & 9.2 & IDP1691-rps22a & $134,286,389-143,421,988$ & \\
\hline & MQTL60 & & & 195.06 & 28.19 & 3 & 7 & jpsb596-pco088782b & $150,643,209-153,998,887$ & \\
\hline & MQTL61 & 10 & 52.88 & 129.12 & 31.33 & 3 & 8 & pco072368-TIDP5776 & $85,246,955-92,538,469$ & \\
\hline \multirow{13}{*}{ 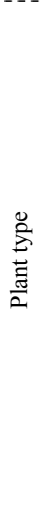 } & MQTL62 & 1 & 53.45 & 334.99 & 9.41 & 3 & 13 & bif2-bnlg1908 & $173,096,051-218,832,513$ & \\
\hline & MQTL63 & & & 405.02 & 7.57 & 3 & 29 & umc2589-umc1500 & $283,187,143-287,192,923$ & \\
\hline & MQTL64 & 2 & 92.29 & 55.93 & 28.87 & 3 & 11.33 & pza02208-nrpd2/e2 & $5,764,847-5,967,363$ & \\
\hline & MQTL65 & & & 101.8 & 4.66 & 6 & 10.83 & wt1-AY110266 & $29,500,958-32,443,663$ & myb49 gpa1 sam2 \\
\hline & MQTL66 & 4 & 75.09 & 123.78 & 6.8 & 3 & 8.33 & isu144b-mmp111 & $15,257,930-17,982,099$ & \\
\hline & MQTL67 & 5 & 88.99 & 166.45 & 8.28 & 3 & 6 & umc2298-dupssr7 & $87,032,692-112,385,161$ & \\
\hline & MQTL68 & & & 210.61 & 8.65 & 4 & 10 & IDP6595-IDP7362 & $173,381,107-155,810,019$ & \\
\hline & MQTL69 & 6 & 65.71 & 325.66 & 9.42 & 6 & 11 & umc238a-uaz81 & $164,260,857-165,096,553$ & \\
\hline & MQTL70 & 8 & 146.96 & 68.68 & 6.63 & 6 & 8.5 & IDP6944-TIDP5457 & $4,805,115-4,918,906$ & \\
\hline & MQTL71 & & & 119.05 & 3.9 & 3 & 11.67 & mmp120-bnlg1067 & $24,558,727-25,351,500$ & rgp2 \\
\hline & MQTL72 & & & 152.36 & 8.47 & 5 & 25.2 & $\operatorname{rap} 2-\lg 4$ & $132,473,577-132,963,880$ & \\
\hline & MQTL73 & 9 & 31.3 & 149.79 & 4.01 & 3 & 9.33 & umc1357-umc1494 & $135,613,607-138,953,873$ & gols3 \\
\hline & MQTL74 & 10 & 37.06 & 123.69 & 5.85 & 4 & 8.5 & umc1938-TIDP5692 & $83,332,319-83,950,011$ & \\
\hline
\end{tabular}

Note. $\mathrm{QTL}=$ quantitative trait loci; $\mathrm{MQTL}=$ meta-QTL; $\mathrm{Chr}=$ chromosome; $\mathrm{AIC}=$ Akaike Information Criterion; $\mathrm{CI}=$ confidence interval; $\mathrm{cm}=$ centi Morgan; $\mathrm{R}^{2}=$ phenotypic variance.

\subsection{Gene Mining With MQTL Intervals Associated With Maize Drought Tolerance}

With the help of flanking marking between MQTL, we observed 9859 genes. MQTL32 of Chr 9 had the most (2 415) number of genes whilst there were no genes in the MQTL13 interval. After sorting out the loci information from the IBM2 2008 Neighbors map, 15991 marker loci were found to confer 4170 genes, among which 1392 genes got annotated functions (Li et al., 2010). In the current study, 118 genes were located within or close to the hot regions detected by meta-analysis. According to functional annotation from the NCBI, 45 gene loci were inferred to 24 hot MQTL regions linked to maize drought tolerance. We downloaded the protein and gene sequences of the hot MQTL regions from the online website PlantGDB. Then, using "Batch search tool" on Pfam website, we analysed the conserved domains of all the sequences. Subsequently, the conserved domain information of these genes was compared with the gene loci information from NCBI. Resultantly, we found out that the gene loci sod4 and gene sequence GRMZM2G169890_T01 of interval MQTL3 had the same conserved domain and the sequence homology ratio of up to $100 \%$.

The conserved domain of taf1 and rps1 in the MQTL12 and MQTL26 were homologous to the gene sequences GRMZM2G002276_T01 and GRMZM5G895282_T01, with sequence homology ratios of $84 \%$ and $87 \%$, respectively. On MQTTL27, three gene loci's (nthr 3 , oc13 and bas 1 ) conserved domains were $73 \%, 100 \%$ and 97\% identical to the gene sequences GRMZM2G073377_T01, GRMZM2G116658_T01 and GRMZM2G077673_T01, respectively. On the MQTL32 interval, there were also three gene loci (apx1, asn4 and pck2) possessing the same conserved domains with the gene sequences GRMZM2G054300_T02, GRMZM2G078472_T01 and GRMZM5G870932_T01, respectively, and sequence homology ratios of $100 \%$, $89 \%$ and $99 \%$, respectively. Besides, the conserved domain of ao1 and kch4 on the MQTL39 and MQTL43 intervals were homologous to the gene sequences GRMZM2G141535_T02 (78\%) and GRMZM2G093313_T02 (99\%), respectively (Table 4). 
Table 4. Gene loci in MQTL intervals possessing identical conserved domains with predicted genes from maize sequence database

\begin{tabular}{llllll}
\hline Trait & MQTL & Chr & Gene loci & Gene ID & Conserved proportion (\%) \\
\hline \multirow{6}{*}{ Mield } & MQTL3 & 1 & sod4 & GRMZM2G169890_T01 & $100 \%$ \\
& MQTL12 & 3 & taf1 & GRMZM2G002276_T01 & $84 \%$ \\
& MQTL26 & 7 & rps1 & GRMZM5G895282_T01 & $87 \%$ \\
& MQTL27 & 7 & nthr3 & GRMZM2G073377_T01 & $73 \%$ \\
& MQTL27 & 7 & oc13 & GRMZM2G116658_T01 & $100 \%$ \\
& MQTL27 & 7 & bas1 & GRMZM2G077673_T01 & $97 \%$ \\
& MQTL32 & 9 & apx1 & GRMZM2G054300_T02 & $100 \%$ \\
& MQTL32 & 9 & asn4 & GRMZM2G078472_T01 & $89 \%$ \\
MQTL32 & 9 & pck2 & GRMZM5G870932_T01 & $99 \%$ \\
& MQTL34 & 10 & nac1 & GRMZM2G015605_T01 & $93 \%$ \\
& MQTL34 & 10 & gst2 & GRMZM2G132093_P01 & $99 \%$ \\
\hline
\end{tabular}

Note. $\mathrm{Chr}=$ chromosome.

\subsection{Extraction of Maize Drought Tolerance Candidate Genes by Gene Comparative Mapping}

By scavenging the Oryza sativa Japonica website GRAMENE, 47 genes were found located on all the 11 Oryza sativa Japonica chromosomes, except on Chr 12. Then, the gene ATG6, located in region 28.070-28.076 cm on Chr 1 of Oryza sativa genetic map IGCN 1998, was transposed onto Chr 8 between the marker rpgc131b and rgpc112 of maize genetic map IBM2008 by 'Cmap' function of the website GRAMENE (Figure 1). Subsequently, the physical map was digged and the Oryza sativa gene was positioned on the region of $13.150-162.498 \mathrm{~cm}$ on the maize physical map B73 RefGen_v2. The online website PlantGDB was used to download predicted gene protein sequences within the range, and the conserved domains were compared with Oryza sativa drought related gene ATG6. Finally, homologous genes GRMZM2G027857 T01 and GRMZM2G027857_T02 on Chr 8 (160,471-160,478 cm) were found (Figure 2). Through NCBI local blast, genes GRMZM2G027857_T01 and GRMZM2G027857_T02 had the same sequence as gene ATG6. It became clear then that gene GRMZ̄M2G027857_T01 and GRMZ̄M2G027857_T02 were candidate genes for response to drought stress in maize. 


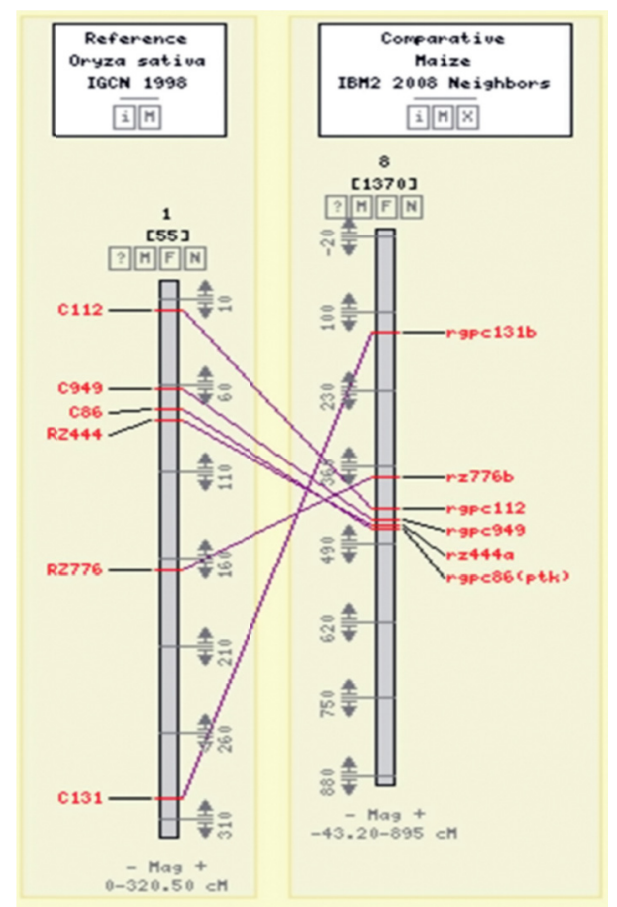

Figure 1. Colinearity comparison of the drought related rice gene ATG6 to maize genes onto an IBM2 Neighbors 2008 map

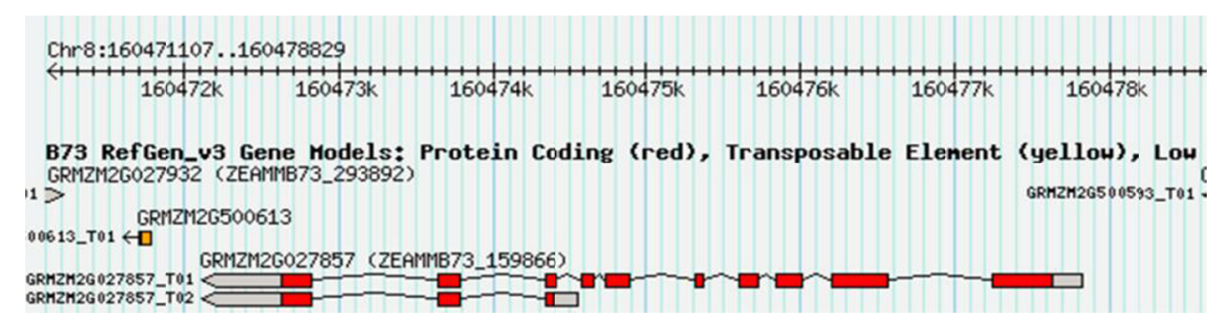

Figure 2. Mapping of maize genes (onto maize B73 RefGen_v3 map) homologous to rice gene ATG6

\section{Discussion}

Drought stress is the major abiotic factor causing a series of complex physiological and biochemical alterations in maize, resulting in yield reduction (Hao et al., 2010). The genetic improvement of maize drought tolerance is therefore critical for sustained food security in the face of the changing global climate. Molecular breeding, encompassing MAS, presents a quicker and better way of improving crop drought tolerance, consequently leading to sustained higher yields (Xu et al., 2014). To date, marker assisted selection has been successfully used in crop genetics and breeding programs in various crops (Aslam et al., 2015).

Efficient molecular breeding scheme entails the dissection of genetic bases underpinning agronomic traits. This involves the correct identification of these traits. The majority of agriculturally important traits are quantitative, meaning that they are controlled by QTL (Wang et al., 2016). Moreover, most important traits are controlled by numerous genes with minor effects. Therefore, an in-depth integration and re-analysis of QTL information is critical for precise location of QTL and quantitative trait genes (QTGs) and understanding the molecular basis underlying these traits and for proper designing of molecular breeding schemes (Blum, 2011; Wang et al., 2016).

Since drought tolerance is best explained as the crop's ability to maintain stable productivity under drought stress, grain yield definitely becomes the final target for selection and breeding for drought tolerance (Hao et al., 2010). QTL mapping of grain yield related traits under drought stress conditions in maize have been widely studied (Lu et al., 2006; Hao et al., 2010; Li et al., 2010). In addition, an array of researches has shown that there is a significant correlation between plant height and drought tolerance under water-limited conditions (Edmeades, 1999; Ziyomo \& Bernardo, 2013; Rex \& Yu, 2017). Further, it is well known that the most vital stage for yield 
loss due to moisture deficit is just before and during flowering; thus flowering date can be an important indicator of drought tolerance (Tuberosa, 2012).

Previously, an array of QTL has been found in maize under drought stress conditions (Veldboom et al., 1994; Frova et al., 1999; Messmer et al., 2009). However, the QTL identified in individual studies usually had too large confidence intervals, which made it difficult to identify the candidate genes related to drought and conduct positional cloning in maize (Arcade et al., 2014). Therefore, integrating QTL data from various researches and narrowing the confidence intervals (CIs) can be essential in eventual QTL cloning and MAS breeding. The meta-analysis methodology proposed by Goffinet and Gerber (2000) has been widely applied in QTL studies. This method is a powerful tool for integrating QTLs detected in different genetic backgrounds and environments and refining QTL position and accuracy, which could be further pursued for MAS or the identification of candidate genes. Peleman and van der Voort (2003) also put forward the technical system of molecular breeding design which suggested that QTL of related agronomic traits should be located first, then allelic variation of these loci evaluated, and lastly, molecular breeding design carried out. This method has been used in integrated QTL of wheat (Zhao et al., 2006), tomato (Sarfatti et al., 1991; Van der Beek et al., 1992), Lactuca sativa (Witsenboer et al., 1995) and maize (Wang et al., 2016).

In the present study, we compiled 457 QTLs related to maize drought tolerance conferring for 18 traits and from 26 previously published researches, and detected 24 MQTs through meta-analysis strategy. As observed by Goffinet and Gerber (2000), the number meta-QTLs and the size of their CIs depend on the initial QTL number, position and CI estimates. Herein our study, the distribution of 443 QTLs under drought stress condition was such that QTL clusters were greatly universal in ten chromosomes. Even our observed MQTLs covered all the chromosomes of the maize genome, this suggesting that maize drought tolerance is a complex quantitative trait controlled by numerous genes with complicated mechanisms (Li et al., 2010). In agreement, Yan et al. (2004) reported that QTLs affecting five vital agronomic traits of maize clustered in chromosomes. Wang et al. (2006) collected 1201 published QTLs affecting 68 traits and imported them into a local CMap software to construct an integrated QTL map, which found that QTL clusters were greatly universal in every chromosomes. In our current study, we observed that QTL clusters existed in chromosomes. For instance, the intervals $200-210 \mathrm{~cm}$ and $220 \mathrm{~cm}-230 \mathrm{~cm}$ on Chr 1 had more than 10 QTLs and these QTLs have been confirmed to control different yield and yield related traits. Tuberosa et al. (2002) made several possible explanations regarding the observed QTL distribution pattern, the most persuasive of which may be one-factor multi-effect or a close linkage between genes that control different traits. Probably, the QTL controlling same traits might not be at random distribution, but there might be QTL concentration on the chromosome that controls a particular trait which might be related to the presence of gene clusters. By comparing the research results from different experiments, here, our finding verifies the universality of QTL cluster distribution. Further, our findings provide a reference for the selection of multi-effect regions in molecular MAS.

Identification and cloning of QTLs can be hampered by imperfect QTL mapping. Those QTLs identified from individual mapping studies usually contain broad confidence interval, where too many genes are mapped and true quantitative trait genes (QTGs) are obscured (Wang et al., 2006). Herein, our integrated maize QTL map had high density of molecular markers from meta-analysis, which clearly reduced the confidence intervals in the MQTL compared with the original maps. The average genetic distance of consistent QTL was $155.05 \mathrm{~cm}$ and there were $32 \%$ MQTL with confidence interval of $<5 \mathrm{~cm}$ which were selected to be hot MQTL. These 'real' QTLs with precise locations were then compared with genes related to drought tolerance in maize and rice, and some candidate genes for drought tolerance were obtained. A total 118 genes were located within or close to the detected hot MQTL regions; and 45 genes from these regions were inferred to 24 maize drought tolerance hot MQTL regions (Table 3).

Predicted genes similar to drought tolerance related genes were identified in many MQTL regions. After comparing the gene loci information (from NCBI) and the gene sequences (from the online website PlantGDB) of these hot MQTL regions, we observed that thirteen gene loci (sod4, taf1, rps1, nthr3, oc13, bas, apx1, asn4, pck2, nacl, gst2, aol and $k c h 4$ ) had the same conserved domains with their corresponding maize gene sequences, with sequence homology ratios ranging from $73 \%$ to $100 \%$ (Table 4). This result confirmed the reliability of MQTLs detected. In agreement, the gene loci fdh2, pep1, phyB2, and nacl, along with the gene of gst and rps family were related to maize drought-tolerance QTLs (Li et al., 2010). This confirms that our meta-analysis can be relied upon and the hot MQTL regions identified herein can be useful in QTG cloning.

However, not all maize genes were overlapped with 'real' QTLs. This could be explained by the fact that some maize genes affecting morphology were not mapped onto IBM2 2008 Neighbors map. Additionally, QTLs with small effect are concealed by mapping populations with lower detection power and 'overview' analysis that tend 
to exclude QTLs with small effect (Wang et al., 2006). To address this, the combination of meta-analysis within a species of interest and synteny-based projections from a related model plant could be an effective way of identifying new candidate genes for trait variation (Chardon et al., 2004).

Our gene comparative mapping analysis identified 47 maize drought tolerance related genes that were located on 11 Oryza sativa Japonica chromosomes, except on Chr 12. By transposing the rice gene ATG6 (located between 28.070-28.076 cm on Chr 1 of Oryza sativa IGCN 1998 genetic map), onto Chr 8 (between rpgc131b and rgpc112 markers) of maize IBM2008 genetic map, we observed that the rice gene was located between $13.150-162.498 \mathrm{~cm}$ on the B73 RefGen_v3 maize physical map (Figure 2). Subsequently, analysis using bioinformatics tools identified that $A T G 6$ is homologous to maize genes GRMZM2G027857 T01 and GRMZM2G027857 T02 on Chr 8 (Figure 2), and that these two were candidate genes for maize drought tolerance. This validates that the employment of a comparative genomics approach in QTL meta-analysis improves the power to detect promising MQTLs and mining out candidate genes underlying drought stress tolerance as has already been reported by previous researchers (Hao et al., 2010; Swamy et al., 2011; Wang et al., 2016).

\section{Conclusion}

In summary, our meta-analysis using 457 original QTLs from diverse studies, conferring 18 traits for yield, plant morphology and flowering time under drought stress in maize, detected 24 MQTLs through the integration of these original QTLs. A total of 47 drought related gene loci were identified and several candidate genes of the hot MQTL were reorganized by bioinformatics techniques. Thirteen gene loci of hot MQTL regions were homologous to their corresponding gene sequences from the PlantGDB database. Further, we used a comparative genomics approach to identify the homologous regions of MQTL in rice database and observed that drought-related rice gene ATG6 was homologous to maize candidate genes GRMZM2G027857_T01 and GRMZM2G027857_T02. The identified hot MQTL regions with narrowed CIs and the candidate genes identified in the current study can serve as valuable reference for MAS and discovering novel genomic segments conferring drought tolerance, and cloned, respectively, to further unravel the molecular mechanisms underpinning maize drought tolerance.

\section{Acknowledgements}

This research was supported by the National Key Research and Development Project of China (Selection and Efficient Combination Model of Wheat and Maize Water Saving, High Yield and High Quality Varieties) [Grant No. 2017YFD0300901].

\section{References}

Adebayo, M. A., Menkir, A., Blay, E., Gracen, V., \& Danquaha, E. Y. (2014). Performance-based grouping of adapted and exotic drought-tolerant maize (Zea mays L) inbred lines under stressed and non-stressed conditions. Maydica, 59(1-4), 116-124.

Agrama, H. A. S., \& Moussa, M. E. (1996). Mapping QTLs in breeding for drought tolerance in maize (Zea mays L.). Euphytica, 91(1), 89-97. https://doi.org/10.1007/BF00035278

Arcade, A., Labourdette, A., Falque, M., Mangin, B., Chardon, F., Charcosset, A., \& Joets, J. (2014). BioMercator: Integrating genetic maps and QTL towards discovery of candidate genes. Bioinformatic, 20(14), 2324-2326. https://doi.org/10.1093/bioinformatics/bth230

Aslam, M., Maqbool, M. A., \& Cengiz, R. (2015). Drought stress in maize (Zea mays L.): Effects, resistance mechanisms, global achievements and biological strategies for improvement. Springer Briefs in Agriculture, 51-54. https://doi.org/10.1007/978-3-319-25442-5

Blum, A. (2011). Plant breeding for water-limited environments. Springer, New York. https://doi.org/10.1007/ 978-1-4419-7491-4

Chardon, F., Virlon, B., Moreau, L., Moreau, L., Falque, M., Joets, J., ... Charcosset, A. (2004). Genetic architecture of flowering time in maize as inferred from quantitative trait loci meta-analysis and synteny conservation with rice genome. Genetics, 68, 2169-2185. https://doi.org/10.1534/genetics.104.032375

Chen, Z. H., Cao, Z. Y., Tian, B., \& Li, L. (2012). Mapping QTL for several drought related traits in maize (Zea mays L.) under field condition. Journal of North China Agriculture, 27, 79-86.

Courtois, B., Ahmadi, N., Khowaja, F., Price, A. H., Rami, J. F., Frouin, J., ... Ruiz, M. (2009). Rice root genetic architecture: Meta-analysis from a QTL database improves resolution to a few candidate genes. Rice, 2, 115-128. https://doi.org/10.1007/s12284-009-9028-9 
Danan, S., Veyrieras, J. B., \& Lefebvre, V. (2011). Construction of a potato consensus map and QTL meta-analysis offer new insights into the genetic architecture of late blight resistance and plant maturity traits. Biogeochmistry, 11, 1-16. https://doi.org/10.1186/1471-2229-11-16

Darvasi, A., \& Soller, M. (1997). A simple method to calculate resolving power and confidence interval of QTL map location. Behav Genet, 27(2), 125-132. https://doi.org/10.1023/A:1025685324830

Edmeades, G. O. (2013). Progress in achieving and delivering drought tolerance in maize-An update. ISAAA: Ithaca NYUSA, 1-39. https://doi.org/ 10.1007/s10118-010-8208-9

Edmeades, G. O., Bolanos, J., Chapman, S. C., Lafitte, H. R., \& Banziger, M. (1999). Selection improves drought tolerance in tropical maize populations: I. Gains in biomass, grain yield, and harvest index. Crop Sci., 39(5), 1306-1315. https://doi.org/10.2135/cropsci1999.3951306x

Frova, C., Krajewski, P., di Fonzo, N., Villa, M., \& Sari-Gorla, M. (1999). Genetic analysis of drought tolerance in maize by molecular markers I. Yield components. Theor. Appl. Genet., 99(12), 280-288. https://doi.org/ $10.1007 / \mathrm{s} 001220051233$

Fu, F. L., Feng, Z. L., Gao, S. B., Zhou, S. F., \& Li, W. C. (2008). Evaluation and Quantitative inheritance of several drought-relative traits in maize. Agricultural Sciences in China, 7(3), 280-290. https://doi.org/ 10.1016/S1671-2927(08)60067-X41

Gao, S. B. (2004). Mapping QTL of traits related to drought tolerance in maize (Unpublished doctoral dissertation. Sichuan Agricultural University, Ya An City, China).

Gao, S. B., Feng, Z. L., Li, W. C., \& Rong, T. Z. (2005a). Mapping QTLs for root and yield under drought stress in maize. Acta Agronomica Sinica, 31, 718-722.

Gao, S. B., Zhao, M. J., Pan, G. T., Li, W. C., \& Rong, T. Z. (2005b). Identification of QTLs controlling flowering parameters of maize under drought stress and non-stress environment. Southwest China Journal of Agricultural Sciences, 18, 593-597.

Goffinet, B., \& Gerber, S. (2000). Quantitative trait loci: A meta-analysis. Genetics, 155(1), $463-473$. https://doi.org/10.1002/1526-968X(200005)27:1<32::AID-GENE50>3.0.CO;2-T

Golam, F., Farhana, N., Zain, M. F., Majid, N. A., Rahman, M. M., Rahman, M. M., \& Kadir, M. A. (2011). Grain yield and associated traits of maize (Zea mays L.) genotypes in Malaysian tropical environment. Afr. J. Agric. Res., 6(28), 6147-6154. https://doi.org/10.5897/AJAR 11.1331

Hall, A. J., Vilella, F., Trapani, N., \& Chimenti, C. (1982). The effects of water stress and genotype on the namics of pollen-shedding and bilking in maize. Field Crop Res., 5, 349-363. https://doi.org/10.1016/ 0378-4290(82)90036-3

Hanocq, E., Laperche, A., Jaminon, O., Lainé, A. L., \& Le, G. J. (2007). Most significant genome regions involved in the control of earliness traits in bread wheat, as revealed by QTL meta-analysis. Theor Appl Genet, 114(3), 569-584. https://doi.org/10.1007/s00122-006-0459-z

Hao, Z. F., Li, X. H., Liu, X. L., Xie, C. X., Li, M. S., Zhang, D. G., \& Zhang, S. H. (2010). Meta-analysis of constitutive and adaptive QTL for drought tolerance in maize. Euphytica, 174(2), 165-177. https://doi.org/ 10.1007/s 10681-009-0 091-5

Hao, Z. F., Li, X. H., Xie, C. X., Li, M .S., Zhang, D. G., Bai, L., \& Zhang, S. H. (2017). Two consensus quantitative trait loci clusters controlling anthesis-silking interval, ear setting and grain yield might be related with drought tolerance in maize. Ann Appl Biol, 153(1), 73-83. https://doi.org/10.1111/ j.1744-7348.2008.00239.x

Jiang, L. L. (2006). QTL analysis of plant height and yield in maize under water stress and normal irrigation condition (Unpublished master's thesis, Inner Mongolia Agricultural University, Hu He Hao Te City, China).

Khairallah, M. M., Bohn, M., Jiang, C., Deutsch, J. A., Jewell, D. C., Mihm, J. A., ... Hoisington, D. A. (1998). Molecular mapping of QTL for southwestern corn borer resistance, plant height and flowering in tropical maize. Plant Breed, 117(4), 309-318. https://doi.org/10.1111/j.1439-0523.1998.tb01947.x

Lacape, J. M., Llewellyn, D., Jacobs, J., Arioli, T., Becker, D., Calhoun, S., ... Viot, C. (2010) Meta-analysis of cotton fiber quality QTLs across diverse environments in a Gossypium hirsutum, $\times \mathrm{G}$ barbadense RIL population. BMC Plant Biol, 10, 132-155. https://doi.org/10.1186/1471-2229-10-132 
Li, W. J., Liu, Z. Z., Shi, Y. D., Song, Y. C., Wang, T. Y., Xu, C. W., \& Li, Y. (2010). Detection of consensus genomic region of QTLs relevant to drought-tolerance in maize by QTL meta-analysis and bioinformatics approach. Acta Agron Sin, 36(9), 1457-1467. https://doi.org/10.1016/S1875-2780(09)60072-9

Li, X. H., Liu, X. D., Li, M. S., \& Zhang, S. H. (2003). Identification of quantitative trait loci for anthesis-silking interval and yield components under drought stress in maize. Acta Botanica Sinica, 45(7), 852-857. https://doi.org/10.1023/A:1022289509702

Lu, G. H., Tang, J. H., Yan, J. B., Ma, X. Q., Li, J. S., Chen, S. J., ... Dai, J. R. (2006). Quantitative trait loci mapping of maize yield and its components under different water treatments at flowering time. J Integr Plant Biol, 48(10), 233-1243. https://doi.org/10.1111/j.1672-9072.2006.00289.x

Maazou, A. R. S., Tu, J. L., Qiu, J., \& Liu, Z. Z. (2016). Breeding for drought tolerance in maize (Zea mays L.). Am J Plant Sci, 7, 1858-1870. https://doi.org/10.4236/ajps.2016.714172

Marino, R., Ponnaiah, M., Krajewski, P., Frova, C., Gianfranceschi, L., Pè, M. E., \& Sari-Gorla, M. (2009). Addressing drought tolerance in maize by transcriptional profling and mapping. Mol Genet Genomics, 281(2), 163-179. https://doi.org/10.1007/s00438-008-0401-y

Messmer, R., Fracheboud, Y., Banziger, M., Vargas, M., Stamp, P., \& Ribaut, J. M. (2009). Drought stress and tropical maize: QTL-by-environment interactions and stability of QTLs across environments for yield components and secondary traits. Theor Appl Genet, 119(5), 913-930. https://doi.org/10.1007/ s00122-009-1099-x

Orf, J. H., Chase, K., Jarvik, T., Mansur, L. M., Cregan, P. B., Adler, F. R., \& Lark, K. G. (1999). Genetics of soybean agronomic traits: I. Comparison of three related recombinant inbred populations. Crop Sci, 39 (6), 1642-1651. https://doi.org/10.2135/cropsci 1999.3961642x

Peleman, J. D., \& van der Voort, J. R. (2003). Breeding by design. Trends Plant Sci, 8(7), 330-334. https://doi.org/10.1016/s1360-1385(03)00134-1

Peng, B., Wang, Y., Li, Y. X., Liu, C., Zhang, Y., Liu, Z. Z., Tan, W. W., ... Li, Y. (2011). QTL Analysis for yield components and kernel-related traits in maize under different water regimes. Acta Agronomica Sinica, 36(11), 1832-1842. https://doi.org/10.3724/SP.J.1006.2010.01832

Rao, G. J. N., Reddy, J. N., Variar M., \& Mahender, A. (2016). Molecular breeding to improve plant breeding to improve plant resistance to abiotic stresses, Advances in Plant Breeding Strategies: Agronomic, Abiotic and Biotic Stress Traits, 283-326. https://doi.org/10.1007/978-3-319-22518-0_8

Rex, B., \& Yu, J. M. (2017). Prospects for genome-wide selection for quantitative traits in maize. Crop Sci, 47(3), 1082-1090. https://doi.org/10.2135/cropsci2006.11.0690

Ribaut, J. M., Betran, J., Monneveux, P. \& Setter, T. (2009). Drought tolerance in maize. In J. L. Hake (Ed.), Handbook of Maize: Its Biology (pp. 311-344). Springer, New York, NY. https://doi.org/10.1007/ 978-0-387-79418-1_16

Ribaut, J. M., Hoisington, D. A., Deutsch, J. A., Jiang, C., \& Gonzalez-de-Leon, D. (1996). Identification of quantitative trait loci under drought conditions in tropical maize. 1. Flowering parameters and the anthesis silking interval. Theor Appl Genet, 92(7), 905-914. https://doi.org/10.1007/BF00221905

Ribaut, J. M., Jiang, C., Gonzalez-de-Leon, D., Edmeades, G .O., \& Hoisington, D. A. (1997). Identification of quantitative trait loci under drought conditions in tropical maize. 2. Yield components and marker-assisted selection strategies. Theor Appl, 94(67), 887-896. https://doi.org/10.1007/s001220050492

Rong, J., Feltus, F. A., Waghmare, V. N., Pierce, G. J., Chee, P. W., Draye, X., ... Paterson, A. H. (2007). Meta-analysis of polyploidy cotton QTL shows unequal contributions of subgenomes to a complex network of genes and gene clusters implicated in lint fiber development. Genetics, 176(4), 2577-2588. https://doi.org/10.1534/genetics.107.074518

Sarfatti, M., Abu-Abied, M., Katan, J., \& Zamir, D. (1991). RFLP mapping of I1, a new locus in tomato conferring resistance against Fusarium oxysporumf sp. lycopersici race 1. Theor Appl Genet, 82(1), 22-26. https://doi.org/10.1007/bf00231273

Sari-Gorla, M., Krajewski, P., Di Fonzo, N., Villa, M., \& Frova, M. (1999). Genetic analysis of drought tolerance in maize by molecular markers. II. Plant height and flowering. Theor Appl Genet, 99(12), 289-295. https://doi.org/10.1007/s001220051234 
Semagn, K., Beyene, Y., Warburton, M. L., Tarekegne, A., Mugo, S., Meisel, B., ... Prasanna, B. M. (2013). Meta-analyses of QTL for grain yield and anthesis silking interval in 18 maize populations evaluated under water-stressed and well-watered environments. BMC Genomics, 14, 313. https://doi.org/10.1186/1471-21 64-14-313

Shin, S., Lee, J. S., Kim, S. G., Go, T. H., Shon, J. Y., Kang, S. G., ... Woo, M. O. (2015). Yield of maize (Zea mays L.) logistically declined with increasing length of the consecutive visible wilting days during flowering. J Crop Sci Biotechnol, 18(4), 237-248. https://doi.org/10.1007/s12892-015-0112-y

Swamy, B. M., Vikram, P., Dixit, S., Ahmed H. U., \& Kumar, A. (2011). Meta-analysis of grain yield QTL identified during agricultural drought in grasses showed consensus. BMC Genomics, 12(1), 319-337. https://doi.org/10.1186/1471-2164-12-319

Truntzler, M., Barriere, Y., Sawkins, M. C., Lespinasse, D., Betran, J., Charcosset, A., \& Moreau, L. (2010). Meta-analysis of QTL involved in silage quality of maize and comparison with the position of candidate genes. Theor Appl Genet, 121, 1456-1482. https://doi.org/10.1007/s00122-010-1402-x

Tuberosa, R. (2012). Phenotyping for drought tolerance of crops in the genomics era. Front Physiol, $3,347$. https://doi.org/10.3389/fphys.2012.00347

Tuberosa, R., Salvi, S., Sanguineti, M. C., Landi, P., Maccaferri, M., \& Conti, S. (2002). Mapping QTL regulating morpho-physiological traits and yield: Case studies, shortcomings and perspectives in drought-stressed maize. Ann Bot, 89(7), 941-963. https://doi.org/10.1093/aob/mcf134

Van der Beek, J. G., Verkerk, R., Zabel, P., \& Lindhout, P. (1992). Mapping strategy for resistance genes in tomato based on RFLPs between cultivars: $C f 9$ (resistance to Cladosporium fulvum) on chromosome 1 . Theor Appl Genet, 84, 106-112.

Veldboom, L. R., \& Lee, M. (1996). Genetic Mapping of Quantitative Trait Loci in Maize in Stress and Nonstress Environments: I. Grain Yield and Yield Components. Crop Sci, 36(5), 1320-1327. https://oi.org/10.2135/cropsci1996.0011183X003600050040x

Veldboom, L. R., Lee, M., \& Woodman, W. L. (1994). Molecular marker-facilitated studies in an elite maize population: I. Linkage analysis and determination of QTL for morphological traits. Theor Appl Genet, 88(1), 7-16. https://doi.org/ 10.1007/BF00222387

Wang, J. Y., Xiong, Y. C., Li, F. M., Siddique, K. H. M., \& Tumer, N. C. (2017). Effects of drought stress on morphophysiological traits, biochemical characteristics, yield and yield components in different ploidy wheat: a meat-analysis. Adv Agron, 143, 139-173. https://doi.org/10.1016/bs.agron.2017.01.002

Wang, X. P. (2011). Analysis of QTL mapping and yield-related traits for drought resistance in maize (Unpublished master's thesis, Shandong Agricultural University, Tai An City, China).

Wang, Y., Xu, J., Deng, D., Ding, H., Bian, Y., Yin, Z., ... Zhao, Y. (2016). A comprehensive meta-analysis of plant morphology, yield, stay-green, and virus disease resistance QTL in maize (Zea mays L.). Planta, 243(2), 459-471. https://doi.org/10.1007/s00425-015-2419-9

Wang, Y., Yao, J., Zhang, Z. F., \& Zheng, Y. L. (2006). The comparative analysis based on maize integrated QTL map and meta-analysis of plant height QTLs. Chin Sci Bull, 51(18), 2219-2230. https://doi.org/ $10.1007 / \mathrm{s} 11434-006-2119-8$

Wang, Y. (2008). QTL analysis of drought tolerance at seedling stage based on introgression lines and F2:3 families of maize (Unpublished doctoral dissertation, Chinese Academy of Agricultural Sciences, Beijing, China).

Witsenboer, H., Kesseli, R. V., Fortin, M. G., Stanghellini, M., \& Michelmore, R. W. (1995). Sources and genetic structure of a cluster of genes for resistance to three pathogens in lettuce. Theor Appl Genet, 91(1), 178-188. https://doi.org/10.1007/bf00220875

Wu, J. W. (2005). Analysis QTL and agronomy traits and genetic diversity of drought tolerance in maize (Unpublished master's thesis, Hebei Agricultural University, Baoding City, China).

Xiang, K., Reid, L. M., Zhang, Z. M., Zhu, X. M., \& Pan, G. T. (2012). Characterization of correlation between grain moisture and ear rot resistance in maize by QTL meta-analysis. Euphytica, 183(2), 185-195. https://doi.org/10.1007/s10681-011-0440-z

Xiao, Y. N. (2015). Genetic analysis and identification of quantitative trait loci (QTLs) for drought tolerance in maize (Zea mays L.) (Unpublished doctoral dissertation. Huazhong Agricultural University, Wuhan City, 
China).

Xu, J., Yuan, Y. B., Xu, Y. B., Zhang, G. Y., Guo, X. S., Wu, F. K., ... Cao, M. L. (2014). Identification of candidate genes for drought tolerance by whole-genome resequencing in maize. Biogeochemistry, 14, 83-98. https://doi.org/10.1186/1471-2229-14-83

Yan, J. B., Tang, H., Huang, Y. Q., Zheng, Y. L., \& Li, J. S. (2004). Comparative analysis of QTL for important agronomic traits between maize and rice. Acta Genet Sin, 31, 1401-1407. https://doi.org/10.1300/ J064v24n01 09

Zhao, H. X., Liu, X. M., \& Chen, M. S. (2006). H22, a major resistance gene to the Hessian fly (Mayetiola destructor), is mapped to the distal region of wheat chromosome 1DS. Theor Appl Genet, 113(8), 1491-1496. https://doi.org/10.1007/s00122-006-0396-X

Zhao, X. Q., Peng, Y. L., Zhang, J. W., Fang, P., \& Wu, B. Y. (2017). Mapping QTLs and meta-QTLs for two inflorescence architecture traits in multiple maize populations under different watering environments. Mol Breed, 37(7), 91. https://doi.org/10.1007/s11032-017-0686-9

Zhu, J. J. (2008). Identification and analysis of quantitative trait loci for drought tolerance in maize (Zea mays L.) (Unpublished master`s thesis, Shandong Agricultural University, Tai An City, China).

Zhu, J. J., Wang, X. P., Sun, C. X., Zhu, X. M., Li, M., Zhang, G. D., ... Wang Z. L. (2011). Mapping of QTL associated with drought tolerance in a semi-automobile rain shelter in maize (Zea mays L.). Agricultural Sciences in China, 10(7), 987-996. https://doi.org/10.1016/S1671-2927(11)60085-0

Ziyomo, C., \& Bernardo, R. (2013). Drought tolerance in maize: Indirect selection through secondary traits versus genomewide selection. Crop Sci, 53(4), 1269-1275. https://doi.org/10.2135/cropsci2012.11.0651 


\section{Appendix A}
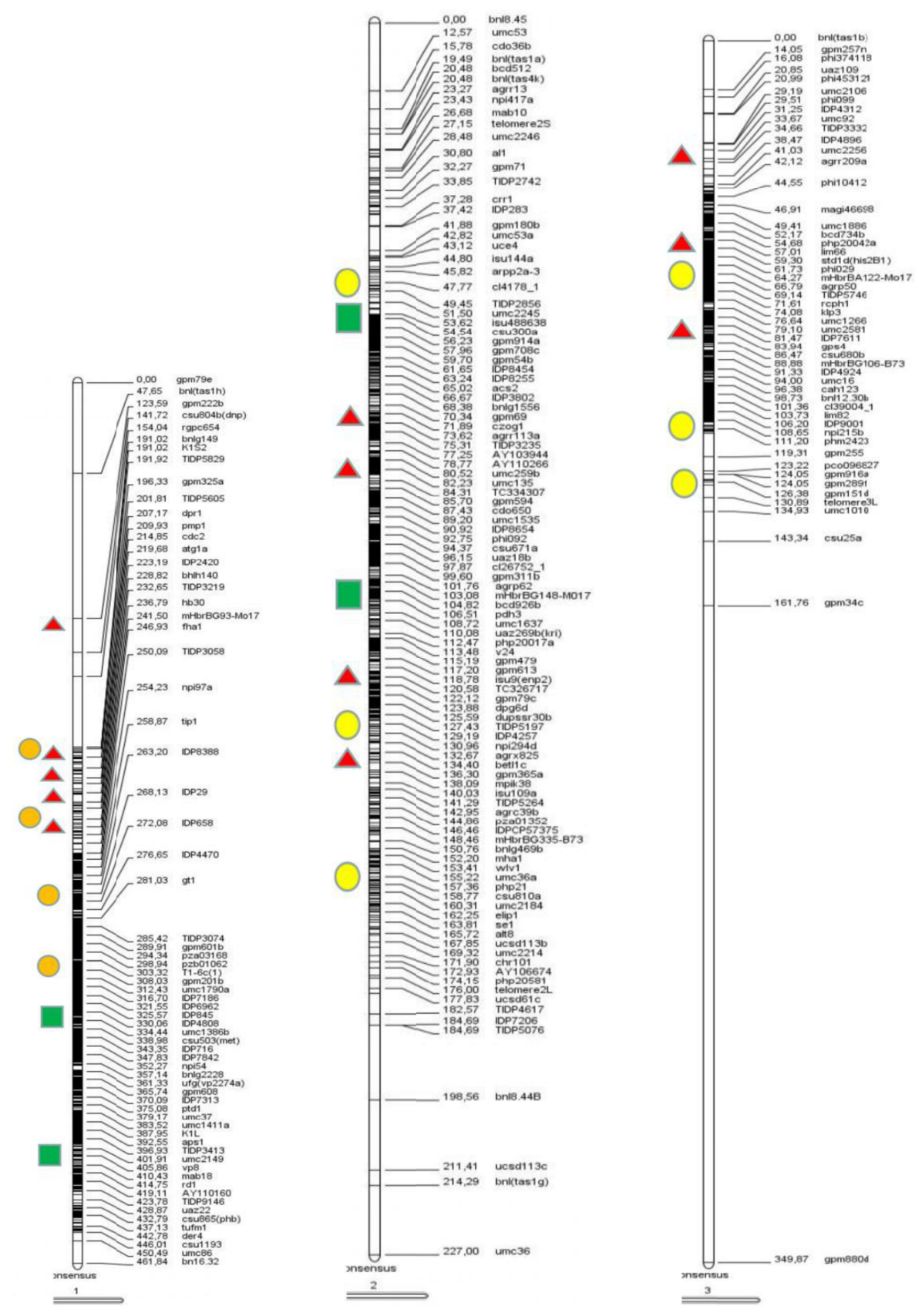

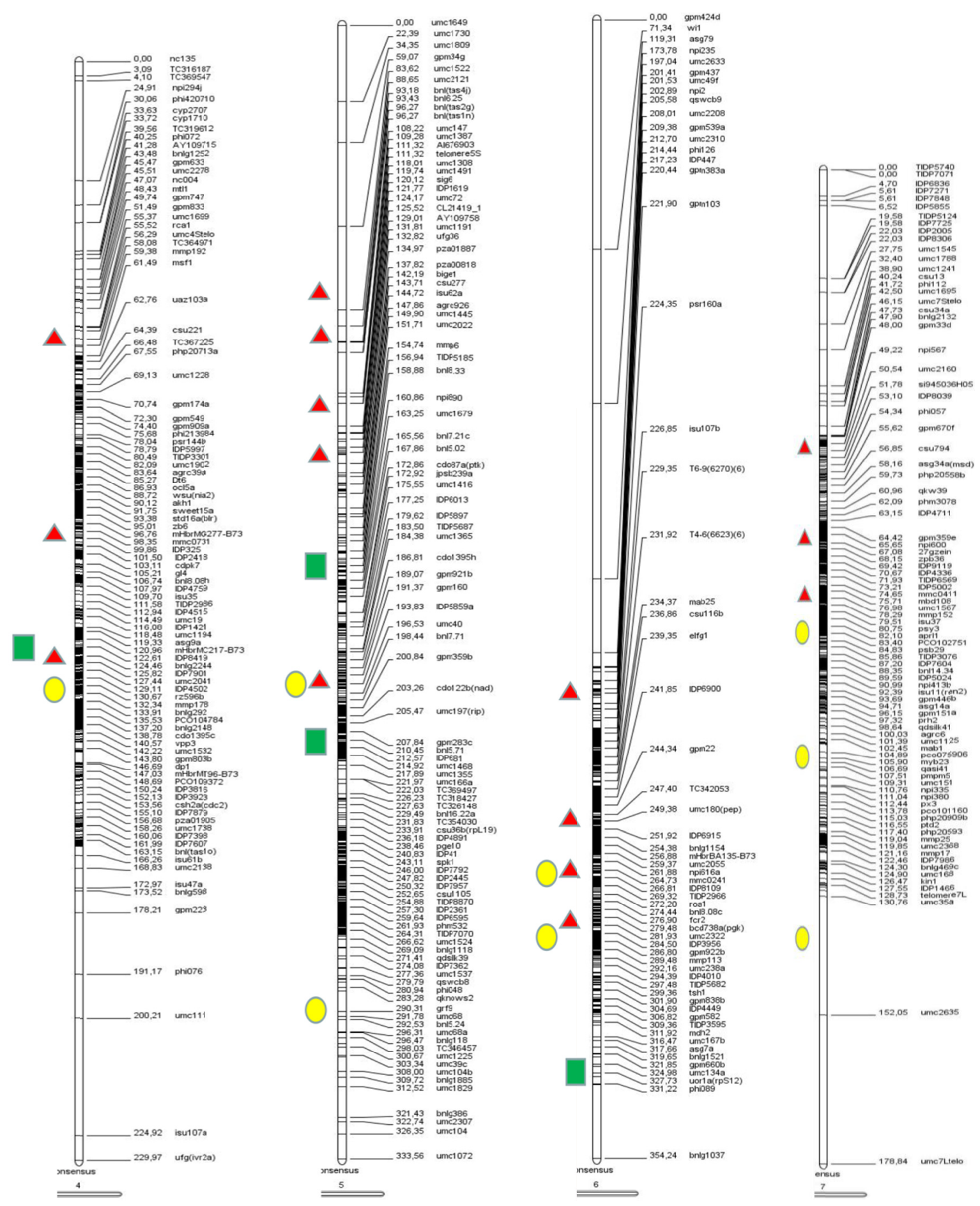

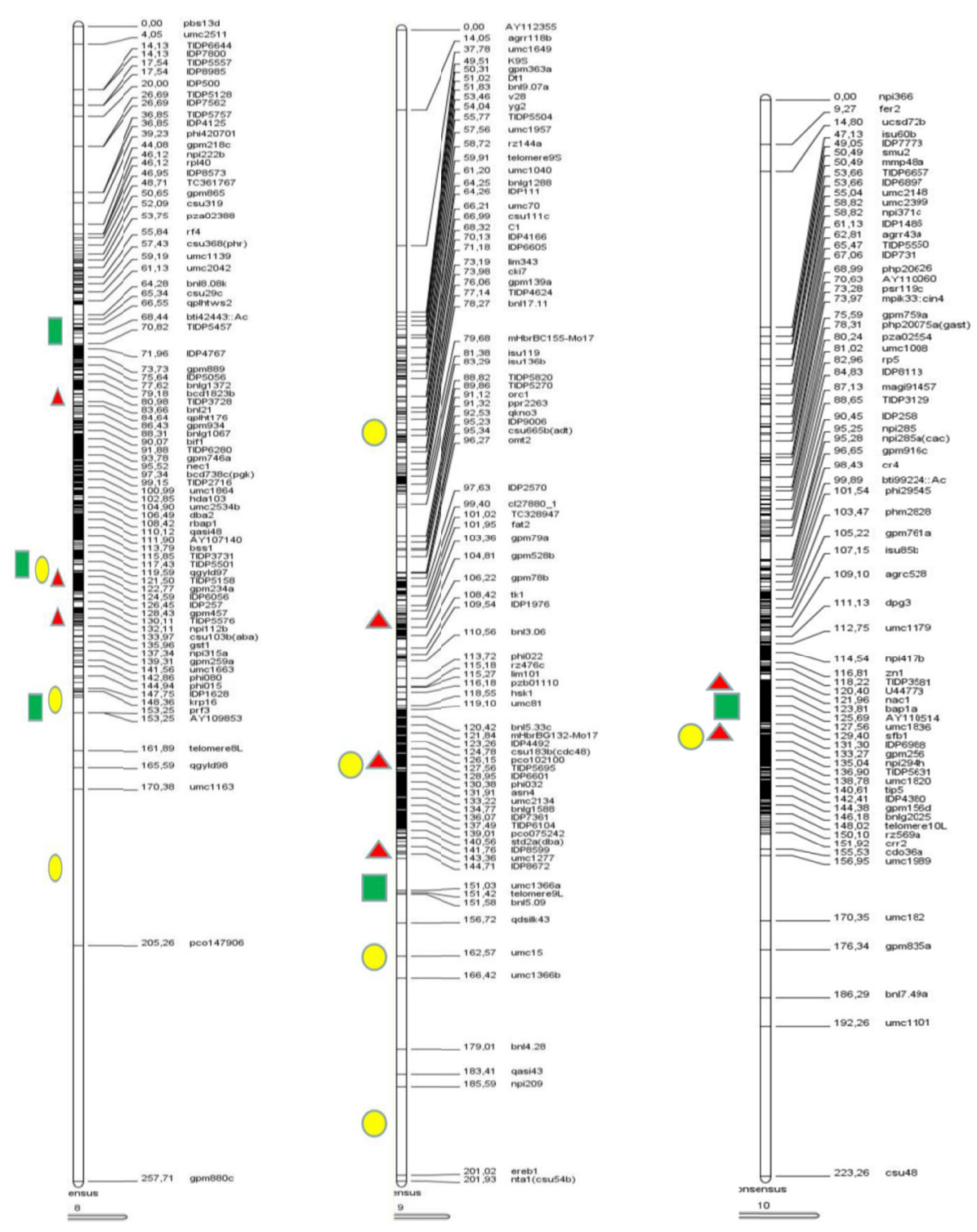

Figure A1. MQTLs revealed by meta-analysis of reported grain yield-, flowering date- and plant type related traits under drought stress conditions, on maize chromosomes, from 1 to 10. Red triangles represent MQTLs related to grain yield, yellow circles represent MQTLs related to flowering date, whereas green squares represent MQTLs related to plant height

\section{Copyrights}

Copyright for this article is retained by the author(s), with first publication rights granted to the journal.

This is an open-access article distributed under the terms and conditions of the Creative Commons Attribution license (http://creativecommons.org/licenses/by/4.0/). 